\title{
Solutions of Nabla Fractional Difference Equations Using N-Transforms
}

\author{
J. Jagan Mohan • G. V. S. R. Deekshitulu
}

Received: 29 July 2013 / Revised: 7 November 2013 / Accepted: 10 March 2014 /

Published online: 25 April 2014

(C) School of Mathematical Sciences, University of Science and Technology of China and Springer-Verlag Berlin Heidelberg 2014

\begin{abstract}
In the present paper, we present some important properties of $\mathrm{N}$-transform, which is the Laplace transform for the nabla derivative on the time scale of integers (Bohner and Peterson in Dynamic equations on time scales, Birkhauser, Boston, 2001; Advances in dynamic equations on time scales, Birkhauser, Boston, 2002). We obtain the $\mathrm{N}$-transform of nabla fractional sums and differences and then apply this transform to solve some nabla fractional difference equations with initial value problems. Finally, using N-transforms, we prove that discrete Mittag-Leffler function is the eigen function of Caputo type nabla fractional difference operator $\nabla^{\alpha}$.
\end{abstract}

Keywords Fractional difference - Caputo type - Exponential order · N-transform · Discrete Mittag-Leffler function

Mathematical Subject Classification (2010) 39 A10 39 399

\section{An Introduction to Nabla Discrete Fractional Calculus}

Fractional calculus has gained importance during the past three decades due to its applicability in diverse fields of science and engineering [1]. The analogous theory for nabla discrete fractional calculus was initiated and properties of the theory of fractional

\footnotetext{
J. Jagan Mohan $(\varangle)$

Department of Mathematics, Birla Institute of Technology and Science Pilani, Hyderabad Campus, Hyderabad 500078, Andhra Pradesh, India e-mail: j.jaganmohan@hotmail.com

G. V. S. R. Deekshitulu

Department of Mathematics, JNTU Kakinada, Kakinada 533003, Andhra Pradesh, India e-mail: dixitgvsr@hotmail.com
} 
sums and differences were established [2,3]. Recently, a series of papers continuing this research has appeared [4-20].

In this section, we introduce some basic definitions and results concerning nabla discrete fractional calculus. Throughout the article, for notations and terminology we refer [21]. The extended binomial coefficient $\left(\begin{array}{l}a \\ n\end{array}\right),(a \in \mathbb{R}, n \in \mathbb{Z})$ is defined by

$$
\left(\begin{array}{l}
a \\
n
\end{array}\right)= \begin{cases}\frac{\Gamma(a+1)}{\Gamma(a-n+1) \Gamma(n+1)} & n>0 \\
1 & n=0 \\
0 & n<0\end{cases}
$$

Definition 1.1 For any complex numbers $\alpha$ and $\beta,(\alpha)_{\beta}$ be defined as follows.

$$
(\alpha)_{\beta}= \begin{cases}\frac{\Gamma(\alpha+\beta)}{\Gamma(\alpha)} & \text { when } \alpha \text { and } \alpha+\beta \text { are neither zero nor negative integers, } \\ 1 & \text { when } \alpha=\beta=0, \\ 0 & \text { when } \alpha=0, \beta \text { is neither zero nor negative integer, } \\ \text { undefined otherwise. }\end{cases}
$$

Lemma 1.1 For any $a, b \in \mathbb{C}$, the quotient expansion of two gamma functions at infinityis is given by

$$
\frac{\Gamma(n+a)}{\Gamma(n+b)}=n^{a-b}\left[1+\bigcirc\left(\frac{1}{n}\right)\right],(|\arg (n+a)|<\pi,|n| \rightarrow \infty) .
$$

Let $u(n): \mathbb{N}_{0}^{+} \rightarrow \mathbb{R}$ and $m-1<\alpha \leq m$ where $\alpha \in \mathbb{R}^{+}$and $m \in \mathbb{N}_{1}^{+}$.

Definition 1.2 [19] The fractional sum operator of order $\alpha$ is defined as

$$
\nabla^{-\alpha} u(n)=\sum_{j=0}^{n-1}\left(\begin{array}{l}
j+\alpha-1 \\
j
\end{array}\right) u(n-j)=\sum_{j=1}^{n}\left(\begin{array}{l}
n-j+\alpha-1 \\
n-j
\end{array}\right) u(j)
$$

Definition 1.3 [19] The Caputo type fractional difference operator of order $\alpha$ is defined as

$$
\nabla^{\alpha} u(n)=\nabla^{\alpha-m}\left[\nabla^{m} u(n)\right]=\sum_{j=0}^{n-1}\left(\begin{array}{l}
j-\alpha+m-1 \\
j
\end{array}\right) \nabla^{m} u(n-j) .
$$

Now, we simplify the above definition for our convenience as follows.

Corollary 1 The equivalent form of (1.3) is

$$
\nabla^{\alpha} u(n)=\sum_{j=1}^{n}\left(\begin{array}{l}
n-j-\alpha-1 \\
n-j
\end{array}\right) u(j)-\sum_{k=0}^{m-1}\left(\begin{array}{l}
n+k-\alpha-1 \\
n-1
\end{array}\right)\left[\nabla^{k} u(j)\right]_{j=0}
$$


Proof We prove the statement (1.4) using mathematical induction on $m$.

For $m=1$,

$$
\begin{aligned}
\nabla^{\alpha} u(n)= & \sum_{j=0}^{n-1}\left(\begin{array}{l}
j-\alpha \\
j
\end{array}\right) \nabla u(n-j) \\
= & u(n)-\left(\begin{array}{l}
n-\alpha-1 \\
n-1
\end{array}\right) u(0)-\alpha \sum_{j=1}^{n-1} \frac{1}{(j-\alpha)}\left(\begin{array}{l}
j-\alpha \\
j
\end{array}\right) u(n-j) \\
& (\text { using [4] }) \\
= & \sum_{j=0}^{n-1}\left(\begin{array}{l}
j-\alpha-1 \\
j
\end{array}\right) u(n-j)-\left(\begin{array}{l}
n-\alpha-1 \\
n-1
\end{array}\right) u(0) \\
= & \sum_{j=1}^{n}\left(\begin{array}{l}
n-j-\alpha-1 \\
n-j
\end{array}\right) u(j)-\left(\begin{array}{l}
n-\alpha-1 \\
n-1
\end{array}\right) u(0) .
\end{aligned}
$$

The statement is true for $m=1$.

For $m=2$,

$$
\begin{aligned}
\nabla^{\alpha} u(n)= & \sum_{j=0}^{n-1}\left(\begin{array}{l}
j-\alpha+1 \\
j
\end{array}\right) \nabla^{2} u(n-j) \\
= & \sum_{j=0}^{n-1}\left(\begin{array}{l}
j-\alpha+1 \\
j
\end{array}\right) \nabla[\nabla u(n-j)] \\
= & \sum_{j=0}^{n-1}\left(\begin{array}{l}
j-\alpha \\
j
\end{array}\right) \nabla u(n-j)-\left(\begin{array}{l}
n-\alpha \\
n-1
\end{array}\right)[\nabla u(n-j)]_{j=n}(\operatorname{using}(1.5)) \\
= & \sum_{j=0}^{n-1}\left(\begin{array}{l}
j-\alpha-1 \\
j
\end{array}\right) u(n-j)-\left(\begin{array}{l}
n-\alpha-1 \\
n-1
\end{array}\right) u(0)-\left(\begin{array}{l}
n-\alpha \\
n-1
\end{array}\right)[\nabla u(n-j)]_{j=n} \\
& (\mathrm{using}(1.5)) \\
= & \sum_{j=1}^{n}\left(\begin{array}{l}
n-j-\alpha-1 \\
n-j
\end{array}\right) u(j)-\sum_{k=0}^{m-1}\left(\begin{array}{l}
n+k-\alpha-1 \\
n-1
\end{array}\right)\left[\nabla^{k} u(j)\right]_{j=0} .
\end{aligned}
$$

The statement is also true for $m=2$. We assume that the statement is true for $m-1$. Then

$$
\begin{aligned}
\nabla^{\alpha} u(n) & =\sum_{j=0}^{n-1}\left(\begin{array}{l}
j-\alpha+m-2 \\
j
\end{array}\right) \nabla^{m-1} u(n-j) \\
& =\sum_{j=1}^{n}\left(\begin{array}{l}
n-j-\alpha-1 \\
n-j
\end{array}\right) u(j)-\sum_{k=0}^{m-2}\left(\begin{array}{l}
n+k-\alpha-1 \\
n-1
\end{array}\right)\left[\nabla^{k} u(j)\right]_{j=0} .
\end{aligned}
$$


Now we prove the statement is true for $m$. Consider

$$
\begin{aligned}
\nabla^{\alpha} u(n)= & \sum_{j=0}^{n-1}\left(\begin{array}{l}
j-\alpha+m-1 \\
j
\end{array}\right) \nabla^{m} u(n-j) \\
= & \sum_{j=0}^{n-1}\left(\begin{array}{l}
j-\alpha+m-1 \\
j
\end{array}\right) \nabla\left[\nabla^{m-1} u(n-j)\right] \\
= & \sum_{j=0}^{n-1}\left(\begin{array}{l}
j-\alpha+m-2 \\
j
\end{array}\right) \nabla^{m-1} u(n-j)-\left(\begin{array}{l}
n-\alpha+m-2 \\
n-1
\end{array}\right) \\
& {\left[\nabla^{m-1} u(n-j)\right]_{j=n}(\mathrm{using}(1.5)) } \\
= & \sum_{j=1}^{n}\left(\begin{array}{l}
n-j-\alpha-1 \\
n-j
\end{array}\right) u(j)-\sum_{k=0}^{m-1}\left(\begin{array}{l}
n+k-\alpha-1 \\
n-1
\end{array}\right)\left[\nabla^{k} u(j)\right]_{j=0} \\
& (\operatorname{using}(1.6)) .
\end{aligned}
$$

Hence by the principle of mathematical induction on $m$, the statement (1.4) is true for $m \in \mathbb{N}_{1}^{+}$.

\section{N-Transforms and Properties}

Differential equations and difference equations are playing a vital role in every aspect of applied mathematics. Investigation and analysis of differential and difference equations arising in applications led to many deep mathematical problems, and there are so many different techniques in order to solve them. Integral transforms is one such important technique, and Laplace transform is a particular case of integral transform.

Laplace transform is a powerful technique for analyzing linear time-invariant systems such as electrical circuits, harmonic oscillators, optical devices, and mechanical systems. This transformation not only provides a fundamentally different way to understand the behavior of the system, but it also drastically reduces the complexity of the mathematical calculations required to analyze the system. Z-transform, like the Laplace transform, is an indispensable mathematical tool for the design, analysis and monitoring of systems. The z-transform is the discrete-time counter-part of the Laplace transform.

Later Bohner and Peterson [22,23] introduced the Laplace transform for an arbitrary time scale (arbitrary closed subset of reals). Two particular choices of time scales, namely the reals and the integers, yield the concepts of the classical Laplace transform and the classical Z-transform. According to the definition given by Bohner and Peterson [22,23], Laplace transform is the classical Laplace transform on $\mathbb{R}$. R- and Ntransforms are discrete analogs of the Laplace transform on the time scale of integers for the alpha derivative. In particular, R-transform is the discrete transform defined on the time scale of integers for the delta $(\Delta)$ derivative and $\mathrm{N}$-transform is the discrete 
transform defined on the time scale of integers for the nabla $(\nabla)$ derivative. R- and $\mathrm{N}$-transforms are not intended to be the more commonly employed Z-transform.

In this section, we derive some important properties of N-transform. Let $u(n)$ : $\mathbb{N}_{1}^{+} \rightarrow \mathbb{R}$. Atici and Eloe [7] defined the discrete transform (N-transform) by

$$
N[u(n)]=\sum_{j=1}^{\infty} u(j)(1-z)^{j-1}=U(z)
$$

for each $z \in \mathbb{C}$ for which the series converges.

Definition 2.1 A function $u(n)$ is said to be of exponential order $r, r>0$ if there exists a constant $A>0$ such that $|u(n)| \leq A r^{-n}$ for sufficiently large $n$.

The following lemma discusses the convergence of N-transform.

Lemma 2.1 Suppose $u(n)$ is of exponential order $r, r>0$. Then $N[u(n)]$ exists for each $z$ lies inside the open ball $B_{1}(r)$.

Proof Suppose $u(n)$ is of exponential order $r, r>0$. Then there exists some constant $A>0$ and a natural number $m$ such that $|u(n)| \leq A r^{-n}$ for each $n>m$. Now, consider

$$
\begin{aligned}
|N[u(n)]| & \leq \sum_{j=1}^{\infty}|u(j)||1-z|^{j-1} \\
& =\sum_{j=0}^{\infty}|u(j+1)||1-z|^{j} \\
& =\sum_{j=0}^{m}|u(j+1)||1-z|^{j}+\sum_{j=m+1}^{\infty}|u(j+1)||1-z|^{j} \\
& \leq \sum_{j=0}^{m}|u(j+1)|(1-z)^{j}+\sum_{j=m+1}^{\infty} \frac{A}{r^{j+1}}|1-z|^{j} \\
& =\sum_{j=0}^{m}|u(j+1)|(1-z)^{j}+\frac{A}{r} \sum_{j=m+1}^{\infty}\left|\frac{1-z}{r}\right|^{j} \\
& =\sum_{j=0}^{m}|u(j+1)|(1-z)^{j}+\frac{A}{r} \frac{\left|\frac{1-z}{r}\right|^{m+1}}{1-\left|\frac{1-z}{r}\right|} \\
& =\sum_{j=0}^{m}|u(j+1)|(1-z)^{j}+\frac{A}{r^{m+1}}\left[\frac{|1-z|^{m+1}}{r-|1-z|}\right] \\
& <\infty .
\end{aligned}
$$

Thus, if $u(n): \mathbb{N}_{1}^{+} \rightarrow \mathbb{R}$ is of exponential order $r, r>0$, then $N[u(n)]$ exists for each $z \in B_{1}(r)$. 
Remark 1 Clearly, for any $a \in \mathbb{R}$ and $p \in \mathbb{R} \backslash\{1\}$, the generalized nabla exponential function $\hat{\mathrm{e}}_{p}(n, a)=\frac{1}{(1-p)^{n-a}}$ is of exponential order $|1-p|$. Thus, $N\left[\hat{\mathrm{e}}_{p}(n, a)\right]$ exists for each $z \in B_{1}(|1-p|)$ and is given by $\frac{1}{z-p}$. Consequently, we have $N[1]=\frac{1}{z}$.

Now we derive some important properties of N-transforms. Let $u(n), v(n): \mathbb{N}_{1}^{+} \rightarrow$ $\mathbb{R}$ are of exponential orders $r, s>0$, respectively and $a$ and $b$ are any scalars.

Theorem 2.2 (Linearity) For each $z \in B_{1}(\min \{r, s\})$,

$$
N[a u(n)+b v(n)]=a N[u(n)]+b N[v(n)] .
$$

Proof Let $p=\min \{r, s\}$. Since $u(n), v(n)$ are of exponential orders $r, s>0$, respectively, we have $|u(n)| \leq r^{-n}$ and $|v(n)| \leq s^{-n}$ for sufficiently large $n \in \mathbb{N}_{1}^{+}$. Consequently,

$$
\begin{aligned}
|a u(n)+b v(n)| & \leq|a||u(n)|+|b||v(n)| \\
& \leq|a| r^{-n}+|b| s^{-n} \\
& \leq[|a|+|b|] p^{-n}
\end{aligned}
$$

for sufficiently large $n \in \mathbb{N}_{1}^{+}$, implying $a u(n)+b v(n)$ is of exponential order $p$, $p>0$. Thus, from Lemma 2.1, $N[a u(n)+b v(n)]$ exists for each $z \in B_{1}(\min \{r, s\})$. Now consider,

$$
\begin{aligned}
N[a u(n)+b v(n)] & =\sum_{j=1}^{\infty}[a u(j)+b v(j)](1-z)^{j-1} \\
& =a \sum_{j=1}^{\infty} u(j)(1-z)^{j-1}+b \sum_{j=1}^{\infty} v(j)(1-z)^{j-1} \\
& =a N[u(n)]+b N[v(n)] .
\end{aligned}
$$

The following lemma relates the shifted N-transform to the original.

Lemma 2.3 (Shifting Theorem) Let $k \in \mathbb{N}_{0}^{+}$. Then for each $z \in B_{1}(r)$,

$$
N[u(n-k)]=(1-z)^{k} N[u(n)]
$$

and

$$
N[u(n+k)]=(1-z)^{-k}\left[N[u(n)]-u(1)-(1-z)^{1} u(2)-\cdots-(1-z)^{k-1} u(k)\right] .
$$

Proof Consider

$$
N[u(n-k)]=\sum_{j=1}^{\infty} u(j-k)(1-z)^{j-1}
$$


Take $j-k=i$ then $i$ varies from 1 to $\infty$. Then

$$
\begin{aligned}
N[u(n-k)] & =\sum_{i=1}^{\infty} u(i)(1-z)^{i+k-1}=(1-z)^{k} \sum_{i=1}^{\infty} u(i)(1-z)^{i-1} \\
& =(1-z)^{k} N[u(n)] .
\end{aligned}
$$

Now consider

$$
N[u(n+k)]=\sum_{j=1}^{\infty} u(j+k)(1-z)^{j-1}
$$

Take $j+k=i$ then $i$ varies from $k+1$ to $\infty$. Then

$$
\begin{aligned}
N[u(n+k)]= & \sum_{i=k+1}^{\infty} u(i)(1-z)^{i-k-1} \\
= & (1-z)^{-k} \sum_{i=k+1}^{\infty} u(i)(1-z)^{i-1} \\
= & (1-z)^{-k}\left[\sum_{i=1}^{\infty} u(i)(1-z)^{i-1}-u(1)-(1-z)^{1} u(2)\right. \\
& \left.-\cdots-(1-z)^{k-1} u(k)\right] \\
= & (1-z)^{-k}\left[N[u(n)]-u(1)-(1-z)^{1} u(2)-\cdots-(1-z)^{k-1} u(k)\right]
\end{aligned}
$$

Definition 2.2 The convolution of $u(n)$ and $v(n)$ is defined as

$$
u(n) * v(n)=\sum_{m=1}^{n} u(m) v(n-m+1) .
$$

Lemma 2.4 (Convolution Theorem) Let $u(n)$ and $v(n)$ are of exponential order $r, r>$ 0. Then

$$
N[u(n) * v(n)]=N[u(n)] N[v(n)]
$$

Proof In order to prove (2.6), first we show that $u(n) * v(n)$ is of some exponential order. Since $u(n), v(n)$ are of exponential order $r>0$, there exist a natural number $m$ such that $|u(n)| \leq r^{-n}$ and $|v(n)| \leq r^{-n}$ for each $n>m$. Consider 


$$
\begin{aligned}
|u(n) * v(n)| & \leq \sum_{k=1}^{n}|u(k)||v(n-k+1)| \\
& =\sum_{k=1}^{m}|u(k)||v(n-k+1)|+\sum_{k=m+1}^{n}|u(k)||v(n-k+1)| \\
& \leq \sum_{k=1}^{m}|u(k)||v(n-k+1)|+\sum_{k=m+1}^{n} r^{-k} r^{-(n-k+1)} \\
& =\sum_{k=1}^{m}|u(k)||v(n-k+1)|+r^{-(n+1)}(n-m) \\
& \leq \sum_{k=1}^{m}|u(k)||v(n-k+1)|+r^{-(n+1)}(n-1) .
\end{aligned}
$$

If $r>1$, then $(n-1)<r^{n-1}$ for sufficiently large $n \in \mathbb{N}_{1}^{+}$and hence

$$
|u(n) * v(n)|<\sum_{k=1}^{m}|u(k)||v(n-k+1)|+r^{-2} \leq A
$$

for some constant $A>0$, implying $u(n) * v(n)$ is of exponential order one. If $0<r \leq 1$, then for $1 \leq \frac{1}{r}<s,(n-1)<s^{(n-1)}$ for sufficiently large $n \in \mathbb{N}_{1}^{+}$ and hence

$$
|u(n) * v(n)|<\sum_{k=1}^{m}|u(k)||v(n-k+1)|+s^{2 n}<s^{3 n}=\left(\frac{1}{s^{3}}\right)^{-n}<p^{-n}
$$

where $0<p<\frac{1}{s^{3}}<1$. Thus, we have $u(n) * v(n)$ is of exponential order $p$, $0<p<1$. Hence $N[u(n) * v(n)]$ exists for each $z \in \bigcup_{0<r \leq 1} B_{1}(r)=B_{1}(1)$. Now, consider

$$
\begin{aligned}
N[u(n) * v(n)] & =\sum_{j=1}^{\infty}[u(j) * v(j)](1-z)^{j-1} \\
& =\left[\sum_{m=1}^{\infty} u(m)(1-z)^{m-1}\right]\left[\sum_{j=1}^{\infty} v(j-m+1)(1-z)^{j-m}\right] .
\end{aligned}
$$

Take $j-m+1=i$ then $i$ varies from 1 to $\infty$. Then

$$
N[u(n) * v(n)]=\left[\sum_{m=1}^{\infty} u(m)(1-z)^{m-1}\right]\left[\sum_{i=1}^{\infty} v(i)(1-z)^{i-1}\right]=N[u(n)] N[v(n)] .
$$


Lemma 2.5 Let $\alpha \in \mathbb{R} \backslash\{\ldots,-2,-1\}$ and $n \in \mathbb{N}_{1}^{+}$. Then for each $z \in B_{1}(1)$,

$$
N\left[\left(\begin{array}{l}
n+\alpha-2 \\
n-1
\end{array}\right)\right]=\frac{1}{z^{\alpha}}
$$

Proof From Lemma 2.1 we know that if $\left(\begin{array}{l}n+\alpha-2 \\ n-1\end{array}\right)$ is of some exponential order $r, r>0$, then $N\left[\left(\begin{array}{l}n+\alpha-2 \\ n-1\end{array}\right)\right]$ exists for each $z \in B_{1}(r)$. Now we will show that $\left(\begin{array}{l}n+\alpha-2 \\ n-1\end{array}\right)$ is of some exponential order.

First, we assume that $\alpha>0$. Then there exists $m \in \mathbb{N}_{1}^{+}$such that $\alpha \leq m$. For sufficiently large $n \in \mathbb{N}_{1}^{+}$and $0<r \leq \frac{1}{m} \leq 1$, we get

$$
\begin{aligned}
\left(\begin{array}{l}
n+\alpha-2 \\
n-1
\end{array}\right)=\frac{\Gamma(n+\alpha-1)}{\Gamma(n) \Gamma(\alpha)} & =\frac{(-1)^{n-1}}{\Gamma(n)} \frac{\Gamma(1-\alpha)}{\Gamma(1-\alpha-\overline{n-1})} \\
& =\frac{(-1)^{n-1}}{\Gamma(n)}(-\alpha)(-\alpha-1) \cdots(-\alpha-\overline{n-2}) \\
& <\frac{(\alpha)^{n-1}}{\Gamma(n)} \\
& <m^{n} \\
& \leq r^{-n}
\end{aligned}
$$

which implies, $\left(\begin{array}{l}n+\alpha-2 \\ n-1\end{array}\right)$ is of exponential order $r, 0<r \leq 1$. Now, suppose that $\alpha<0$. Using Lemma 1.1, for sufficiently large $n \in \mathbb{N}_{1}^{+}$,

$$
\left(\begin{array}{l}
n+\alpha-2 \\
n-1
\end{array}\right)=\frac{\Gamma(n+\alpha-1)}{\Gamma(n) \Gamma(\alpha)}=\frac{n^{\alpha-1}}{\Gamma(\alpha)} \leq 1
$$

implying $\left(\begin{array}{l}n+\alpha-2 \\ n-1\end{array}\right)$ is of exponential order $r=1$. Thus, we conclude that $\left(\begin{array}{l}n+\alpha-2 \\ n-1\end{array}\right)$ is of exponential order $r, 0<r \leq 1$. Hence $N\left[\left(\begin{array}{l}n+\alpha-2 \\ n-1\end{array}\right)\right]$ exists for each $z \in \bigcup_{0<r \leq 1} B_{1}(r)=B_{1}(1)$. Now, consider

$$
N\left[\left(\begin{array}{l}
n+\alpha-2 \\
n-1
\end{array}\right)\right]=\sum_{j=1}^{\infty}\left(\begin{array}{l}
j+\alpha-2 \\
j-1
\end{array}\right)(1-z)^{j-1}=[1-(1-z)]^{-\alpha}=\frac{1}{z^{\alpha}} .
$$


Lemma 2.6 Suppose $u(n)$ is of exponential order $r, 0<r \leq 1$ and let $\alpha \in \mathbb{R}^{+}$. Then, for each $z \in B_{1}(1)$,

$$
N\left[\nabla^{-\alpha} u(n)\right]=z^{-\alpha} N[u(n)] .
$$

Proof From Lemma 2.1 we know that if $\nabla^{-\alpha} u(n)$ is of some exponential order $s$, $s>0$, then $N\left[\nabla^{-\alpha} u(n)\right]$ exists for all $z \in B_{1}(s)$. Now we will show that $N\left[\nabla^{-\alpha} u(n)\right]$ is of some exponential order. Since $u(n)$ and $\left(\begin{array}{l}n+\alpha-2 \\ n-1\end{array}\right)$ are of exponential order $r, 0<r \leq 1$, from Lemma 2.5,

$$
\nabla^{-\alpha} u(n)=\sum_{j=1}^{n}\left(\begin{array}{l}
n-j+\alpha-1 \\
n-j
\end{array}\right) u(j)=u(n) *\left(\begin{array}{l}
n+\alpha-2 \\
n-1
\end{array}\right)
$$

is also of exponential order $r, 0<r \leq 1$. Now, consider

$$
N\left[\nabla^{-\alpha} u(n)\right]=N\left[u(n) *\left(\begin{array}{l}
n+\alpha-2 \\
n-1
\end{array}\right)\right]=z^{-\alpha} N[u(n)] .
$$

Lemma 2.7 Suppose $u(n)$ is of exponential order $r, 0<r \leq 1$ and let $\alpha \in \mathbb{R}^{+}$, $m \in \mathbb{Z}^{+}$such that $m-1<\alpha<m$. Then for each for each $z \in B_{1}(1)$,

$$
N\left[\nabla^{\alpha} u(n)\right]=z^{\alpha}\left[N[u(n)]-\sum_{k=0}^{m-1} \frac{1}{z^{k+1}}\left[\nabla^{k} u(j)\right]_{j=0}\right] .
$$

Proof From Definition 1.3, the Caputo type fractional difference operator of order $\alpha$ is given by

$$
\nabla^{\alpha} u(n)=\nabla^{-(m-\alpha)}\left[\nabla^{m} u(n)\right]
$$

Since $u(n)$ is of exponential order $r, 0<r \leq 1, \nabla^{m} u(n)$ is also of exponential order $r, 0<r \leq 1$. Then, using Lemma 2.6, we conclude that $\nabla^{\alpha} u(n)$ is of exponential order $r, 0<r \leq 1$. Thus $N\left[\nabla^{\alpha} u(n)\right]$ exists for each $z \in B_{1}(1)$. Consider

$$
\begin{aligned}
N\left[\nabla^{\alpha} u(n)\right] & =N\left[\sum_{j=1}^{n}\left(\begin{array}{l}
n-j-\alpha-1 \\
n-j
\end{array}\right) u(j)-\sum_{k=0}^{m-1}\left(\begin{array}{l}
n+k-\alpha-1 \\
n-1
\end{array}\right)\left[\nabla^{k} u(j)\right]_{j=0}\right] \\
& =N_{1}+N_{2}
\end{aligned}
$$

where

$$
N_{1}=N\left[\sum_{j=1}^{n}\left(\begin{array}{l}
n-j-\alpha-1 \\
n-j
\end{array}\right) u(j)\right]=z^{\alpha} N[u(n)]
$$


and

$$
N_{2}=N\left[\sum_{k=0}^{m-1}\left(\begin{array}{l}
n+k-\alpha-1 \\
n-1
\end{array}\right)\left[\nabla^{k} u(j)\right]_{j=0}\right]=\sum_{k=0}^{m-1} N\left[\left(\begin{array}{l}
n+k-\alpha-1 \\
n-1
\end{array}\right)\right]\left[\nabla^{k} u(j)\right]_{j=0} .
$$

Now we consider

$$
N\left[\left(\begin{array}{l}
n+k-\alpha-1 \\
n-1
\end{array}\right)\right]=\frac{1}{z^{k-\alpha+1}}
$$

Thus

$$
N\left[\nabla^{\alpha} u(n)\right]=z^{\alpha}\left[N[u(n)]-\sum_{k=0}^{m-1} \frac{1}{z^{k+1}}\left[\nabla^{k} u(j)\right]_{j=0}\right] .
$$

Definition 2.3 Let $u(n): \mathbb{N}_{1}^{+} \rightarrow \mathbb{R}$ is of exponential order $r, r>0$. Then $U(z)=$ $\sum_{j=1}^{\infty} u(j)(1-z)^{j-1}$ exists for each $z$ lies inside the open ball $B_{1}(r)$ and the inverse $\mathrm{N}$-transform of $U(z)$ is defined as

$$
N^{-1}(U(z))=u(n)
$$

The following are some important properties of inverse N-transforms.

Theorem 2.8 Let $u(n), v(n): \mathbb{N}_{1}^{+} \rightarrow \mathbb{R}$ are of exponential orders $r, s>0$, respectively and $a$ and $b$ are any scalars such that $N[u(n)]=U(z)$ and $N[v(n)]=V(z)$. Then,

1. $N^{-1}[a U(z)+b V(z)]=a u(n)+b v(n)$, for each $z \in B_{1}(\min \{r, s\})$;

2. $N^{-1}[U(z) V(z)]=u(n) * v(n)$, if $r=s$;

3. Let $\alpha \in \mathbb{R} \backslash\{\ldots,-2,-1\}$. Then for each $z \in B_{1}(1), N^{-1}\left[\frac{1}{z^{\alpha}}\right]=\left(\begin{array}{l}n+\alpha-2 \\ n-1\end{array}\right)$.

\section{Solutions of Fractional Difference Equations Using N-Transforms}

In this section, we will illustrate the possible use of the N-transform by applying it to solve some fractional order initial value problems.

In 2003, Nagai [19] defined the discrete Mittag-Leffler function

$$
F_{\alpha}(a, n)=\sum_{j=0}^{\infty}\left[a^{j}\left(\begin{array}{l}
n+j \alpha-1 \\
n-j
\end{array}\right)\right]
$$

which is a generalization of nabla exponential function on the time scale of integers $[22,23]$. He also proved that $F_{\alpha}(a, n)$ is an eigen function of Caputo type fractional 
difference operator defined in (1.3), that is,

$$
\nabla^{\alpha} F_{\alpha}(a, n)=a F_{\alpha}(a, n)
$$

Now we prove the same using N-transforms.

Example 1 Let $u(n)$ is of exponential order $r, 0<r \leq 1$ and let $\alpha \in \mathbb{R}$ such that $0<\alpha<1$. Then the solution of

$$
\begin{aligned}
\nabla^{\alpha} u(n) & =a u(n), \\
u(0) & =a_{0},
\end{aligned}
$$

is $F_{\alpha}(a, n)$, where $a$ and $a_{0}$ are constants.

Solution Taking N-transforms on both sides of (3.3), we have

$$
\begin{aligned}
z^{\alpha}\left[N[u(n)]-\frac{u(0)}{z}\right] & =a N[u(n)] \\
\text { or } N[u(n)] & =a_{0}\left[\frac{z^{\alpha-1}}{z^{\alpha}-a}\right] \\
\text { or } N[u(n)] & =a_{0}\left[\frac{1}{z}+a z^{-\alpha-1}+a^{2} z^{-2 \alpha-1}+\cdots\right] .
\end{aligned}
$$

Applying inverse N-transforms on both sides, we get

$$
\begin{aligned}
u(n) & =a_{0}\left[1+a\left(\begin{array}{l}
n+\alpha-1 \\
n-1
\end{array}\right)+a^{2}\left(\begin{array}{l}
n+2 \alpha-1 \\
n-2
\end{array}\right)+\cdots\right] \\
& =a_{0} \sum_{j=0}^{\infty}\left[a^{j}\left(\begin{array}{l}
n+j \alpha-1 \\
n-j
\end{array}\right)\right] \\
& =a_{0} F_{\alpha}(a, n) .
\end{aligned}
$$

Remark 2 It is clear from the above example that

$$
N\left[F_{\alpha}(a, n)\right]=\frac{z^{\alpha-1}}{z^{\alpha}-a} .
$$

Example 2 Let $u(n)$ is of exponential order $r, 0<r \leq 1$ and let $\alpha \in \mathbb{R}, m \in \mathbb{Z}^{+}$such that $m-1<\alpha<m$. Find the solution of

$$
\begin{aligned}
\nabla^{\alpha} u(n) & =a u(n), \\
{\left[\nabla^{k} u(j)_{j=0}\right] } & =a_{k}, \quad k=0,1,2, \ldots,(m-1),
\end{aligned}
$$

where $a, a_{0}, a_{1}, \ldots, a_{m-1}$ are constants. 
Solution Taking N-transforms on both sides of (3.6), we have

$z^{\alpha}\left[N[u(n)]-\sum_{k=0}^{m-1} \frac{1}{z^{k+1}}\left[\nabla^{k} u(j)\right]_{j=0}\right]=a N[u(n)]$ or $N[u(n)]=\sum_{k=0}^{m-1} a_{k}\left[\frac{z^{\alpha-k-1}}{z^{\alpha}-a}\right]$.

Applying inverse N-transforms on both sides and then convolution theorem, we get

$$
\begin{aligned}
u(n)=\sum_{k=0}^{m-1} a_{k} N^{-1}\left[\frac{z^{\alpha-k-1}}{z^{\alpha}-a}\right] & =\sum_{k=0}^{m-1} a_{k} N^{-1}\left[\frac{z^{\alpha-1}}{z^{\alpha}-a} \times \frac{1}{z^{k}}\right] \\
& =\sum_{k=0}^{m-1} a_{k}\left[F_{\alpha}(a, n) *\left(\begin{array}{l}
n+k-2 \\
n-1
\end{array}\right)\right] \\
& =\sum_{k=0}^{m-1} a_{k} \sum_{j=1}^{n}\left[F_{\alpha}(a, j) \times\left(\begin{array}{l}
n-j+k-1 \\
n-j
\end{array}\right)\right] \\
& =\sum_{k=0}^{m-1} \sum_{j=1}^{n}\left[a_{k} F_{\alpha}(a, j)\left(\begin{array}{l}
n-j+k-1 \\
n-j
\end{array}\right)\right]
\end{aligned}
$$

Example 3 Let $u(n)$ and $v(n)$ are of exponential order $r, 0<r \leq 1$ and let $\alpha \in \mathbb{R}$ such that $0<\alpha<1$. Find the solution of

$$
\begin{aligned}
\nabla^{\alpha} u(n) & =a v(n), \\
u(0) & =a_{0},
\end{aligned}
$$

where $a$ and $a_{0}$ are constants.

Solution Taking N-transforms on both sides of (3.8), we have

$$
\begin{aligned}
z^{\alpha}\left[N[u(n)]-\frac{u(0)}{z}\right] & =a N[v(n)] \\
\text { or } N[u(n)] & =a_{0}\left[\frac{1}{z}\right]+a\left[N[v(n)] \times \frac{1}{z^{\alpha}}\right] .
\end{aligned}
$$

Applying inverse N-transforms on both sides and then convolution theorem, we get

$$
\begin{aligned}
u(n) & =a_{0}+a N^{-1}\left[N[v(n)] \times \frac{1}{z^{\alpha}}\right] \\
& =a_{0}+a\left[v(n) *\left(\begin{array}{l}
n+\alpha-2 \\
n-1
\end{array}\right)\right] \\
& =a_{0}+\sum_{j=1}^{n}\left[a v(j)\left(\begin{array}{l}
n-j+\alpha-1 \\
n-j
\end{array}\right)\right] .
\end{aligned}
$$


Example 4 Let $u(n)$ and $v(n)$ are of exponential order $r, 0<r \leq 1$ and let $\alpha \in \mathbb{R}$, $m \in \mathbb{Z}^{+}$such that $m-1<\alpha<m$. Find the solution of

$$
\begin{aligned}
\nabla^{\alpha} u(n) & =a v(n), \\
{\left[\nabla^{k} u(j)_{j=0}\right] } & =a_{k}, \quad k=0,1,2, \ldots,(m-1),
\end{aligned}
$$

where $a, a_{0}, a_{1}, \ldots, a_{m-1}$ are constants.

Solution Taking N-transforms on both sides of (3.10), we have

$$
\begin{aligned}
z^{\alpha}\left[N[u(n)]-\sum_{k=0}^{m-1} \frac{1}{z^{k+1}}\left[\nabla^{k} u(j)\right]_{j=0}\right] & =a N[v(n)] \\
\text { or } N[u(n)] & =\sum_{k=0}^{m-1} a_{k}\left[\frac{1}{z^{k+1}}\right]+a\left[N[v(n)] \times \frac{1}{z^{\alpha}}\right] .
\end{aligned}
$$

Applying inverse $\mathrm{N}$-transforms on both sides and then convolution theorem, we get

$$
\begin{aligned}
u(n) & =\sum_{k=0}^{m-1} a_{k} N^{-1}\left[\frac{1}{z^{k+1}}\right]+a N^{-1}\left[N[v(n)] \times \frac{1}{z^{\alpha}}\right] \\
& =\sum_{k=0}^{m-1} a_{k}\left(\begin{array}{l}
n+k-1 \\
n-1
\end{array}\right)+a\left[v(n) *\left(\begin{array}{l}
n+\alpha-2 \\
n-1
\end{array}\right)\right] \\
& =\sum_{k=0}^{m-1} a_{k}\left(\begin{array}{l}
n+k-1 \\
n-1
\end{array}\right)+\sum_{j=1}^{n} a\left[v(j)\left(\begin{array}{l}
n-j+\alpha-1 \\
n-j
\end{array}\right)\right] .
\end{aligned}
$$

Example 5 Let $u(n)$ and $v(n)$ are of exponential order $r, 0<r \leq 1$ and let $\alpha \in \mathbb{R}$ such that $0<\alpha<1$. Find the solution of

$$
\begin{aligned}
\nabla^{\alpha} u(n) & =a u(n)+b v(n), \\
u(0) & =a_{0},
\end{aligned}
$$

where $a, b$ and $a_{0}$ are constants.

Solution Taking N-transforms on both sides of (3.12), we have

$$
\begin{aligned}
z^{\alpha}\left[N[u(n)]-\frac{u(0)}{z}\right] & =a N[u(n)]+b N[v(n)] \\
\text { or } N[u(n)] & =a_{0}\left[\frac{z^{\alpha-1}}{z^{\alpha}-a}\right]+b\left[N[v(n)] \times \frac{1}{z^{\alpha}}\right] .
\end{aligned}
$$


Applying inverse N-transforms on both sides and applying convolution theorem, we get

$$
u(n)=a_{0} F_{\alpha}(a, n)+b \sum_{j=1}^{n}\left[v(j)\left(\begin{array}{l}
n-j+\alpha-1 \\
n-j
\end{array}\right)\right] .
$$

Example 6 Let $u(n)$ and $v(n)$ are of exponential order $r, 0<r \leq 1$ and let $\alpha \in \mathbb{R}$, $m \in \mathbb{Z}^{+}$such that $m-1<\alpha<m$. Find the solution of

$$
\begin{aligned}
\nabla^{\alpha} u(n) & =a u(n)+b v(n), \\
{\left[\nabla^{k} u(j)_{j=0}\right] } & =a_{k}, \quad k=0,1,2, \ldots,(m-1),
\end{aligned}
$$

where $a, b, a_{0}, a_{1}, \ldots, a_{m-1}$ are constants.

Solution Taking N-transforms on both sides of (3.14), we have

$$
\begin{aligned}
z^{\alpha}\left[N[u(n)]-\sum_{k=0}^{m-1} \frac{1}{z^{k+1}}\left[\nabla^{k} u(j)\right]_{j=0}\right] & =a N[u(n)]+b N[v(n)] \\
\text { or } N[u(n)] & =\sum_{k=0}^{m-1} a_{k}\left[\frac{z^{\alpha-k-1}}{z^{\alpha}-a}\right]+b\left[N[v(n)] \times \frac{1}{z^{\alpha}}\right] .
\end{aligned}
$$

Applying inverse N-transforms on both sides and then convolution theorem, we get

$$
\begin{aligned}
u(n) & =\sum_{k=0}^{m-1} a_{k} N^{-1}\left[\frac{z^{\alpha-k-1}}{z^{\alpha}-a}\right]+b N^{-1}\left[N[v(n)] \times \frac{1}{z^{\alpha}}\right] \\
& =\sum_{k=0}^{m-1} \sum_{j=1}^{n}\left[a_{k} F_{\alpha}(a, j)\left(\begin{array}{l}
n-j+k-1 \\
n-j
\end{array}\right)\right]+\sum_{j=1}^{n} b\left[v(j)\left(\begin{array}{l}
n-j+\alpha-1 \\
n-j
\end{array}\right)\right] .
\end{aligned}
$$

Acknowledgments The authors are grateful to the referees for their suggestions and comments which considerably helped to improve the content of the paper.

\section{References}

1. Podlubny, I.: Fractional Differential Equations. Academic Press, San Diego (1999)

2. Gray, H.L., Zhang, N.F.: On a new definition of the fractional difference. Math. Comp. 50, 513-529 (1988)

3. Miller, K.S., Ross, B.: Fractional difference calculus. In: Proceedings of the International Symposium on Univalent Functions, Fractional Calculus and Their Applications, pp. 139-152, Nihon University, Koriyama, Japan (1989)

4. Deekshitulu, G.V.S.R., Jagan Mohan, J.: Fractional difference inequalities. Commun. Appl. Anal. 14(1), 89-98 (2010) 
5. Deekshitulu, G.V.S.R., Jagan Mohan, J.: Acta Et Commentationes Universitatis Tartuensis De Mathematica. Fractional difference inequalities of Gronwall-Bellman type 17(1), 19-30 (2013)

6. Deekshitulu, G.V.S.R., Jagan Mohan, J.: Some New Fractional Difference Inequalities of GronwallBellman type. Math. Sci. doi:10.1186/2251-7456-6-69

7. Atici, F.M., Eloe, P.W.: Discrete fractional calculus with the nabla operator. Electron. J. Qual. Theory Differ. Eqs. 3, 1-12 (2009)

8. Atici, F.M., Eloe, P.W.: Linear systems of nabla fractional difference equations. Rocky Mount. J. Math. 41(2), 353-370 (2011)

9. George Anastassiou, A.: Nabla discrete fractional calculus and nabla inequalities. Math. Comput. Model. 51, 562-571 (2010)

10. Hein, J., Mc Carthy, S., Gaswick, N., Mc Kain, B., Spear, K.: Laplace transforms for the nabla difference operator. PanAm. Math. J. 21(3), 79-96 (2011)

11. Jagan Mohan J.: Solutions of perturbed linear nabla fractional difference equations. In: Differential Equations and Dynamical Systems. Springer, Berlin (2013) doi:10.1007/s12591-013-0179-1

12. Jagan Mohan, J.: Solutions of perturbed nonlinear nabla fractional difference equations. Novi Sad J. Math. (to appear)

13. Mohan Jagan, J., Deekshitulu, G.V.S.R.: Comparison theorems on fractional order difference equations, Proyecciones. J. Math. 31(4), 47-62 (2013)

14. Mohan Jagan, J., Deekshitulu, G.V.S.R.: Difference inequalities of fractional order, Proyecciones. J. Math. 32(3), 199-213 (2013)

15. Jagan Mohan, J., Deekshitulu, G.V.S.R.: Fractional order difference equations. Int. J. Differ. Eqs. 2012(780619), 11. doi:10.1155/2012/780619

16. Čermák, J., Kisela, T.: Note on a discretization of a linear fractional differential equation. Math. Bohem. 135(2), 179-188 (2010)

17. Acar, N., Atici, F.M.: Exponential functions of discrete fractional calculus. Appl. Anal. Discret. Math. 7, 343-353 (2013)

18. Abdeljawad, T., Atici, F.M.: On the definitions of nabla fractional operators. Abst. Appl. Anal. Volume 2012, Article ID 406757, 13. doi:10.1155/2012/406757

19. Atsushi, N.: An integrable mapping with fractional difference. J. Phys. Soc. Jpn. 72, 2181-2183 (2003)

20. Abdeljawad, T.: On Riemann and Caputo fractional differences. Comput. Math. Appl. 62, 1602-1611 (2011)

21. Agarwal, R.P.: Difference Equations and Inequalities. Marcel Dekker, New York (1992)

22. Bohner, M., Peterson, A.: Advances in Dynamic Equations on Time Scales. Birkhauser, Boston (2002)

23. Bohner, M., Peterson, A.: Dynamic Equations on Time Scales. Birkhauser, Boston (2001) 